\title{
Carbon nanotubes for organ regeneration: an electrifying performance
}

\author{
S. Marchesan, ${ }^{1 *}$ S. Bosi, ${ }^{1}$ A. Alshatwi, ${ }^{2}$ and M. Prato ${ }^{1 *}$. \\ ${ }^{1}$ Department of Chemical and Pharmaceutical Sciences, University of Trieste, Piazzale Europa 1, 34127 \\ Trieste, Italy \\ ${ }^{2}$ Department of Food and Nutrition, College of Food and Agriculture Science, King Saudi University, P. O. \\ Box 2460 Riyadh - 11451, Saudi Arabia
}

Highlights:

- Organ regeneration is possible thanks to appropriate use of stem cells

- CNT performance in heart and nerve tissue regeneration is unmatched

- Conductive cells cultured on/in carbon nanostructure-scaffolds are "electrified", i.e., more active

- 3D-printing of tissues is revolutionising therapy and the combined use of stem cells and CNTs is promising for organ printing

Keywords: 3D-printing, carbon nanotubes, stem cell, regeneration

Summary: Tissue regeneration research is advancing rapidly, thanks to the innovation potential of stem cells and nanomaterials. In particular, carbon nanotubes (CNTs) have shown an unmatched performance in conductive tissue regeneration. When grown in contact with CNTs, conducting cells become "electrified", i.e., electrically more active, mature, and better interconnected. The challenges inherent in translating these concepts into 3D printing of whole organs for biomedical use are being addressed worldwide.

The field of tissue regeneration is making rapid progress, thanks to the advancements in both use of stem cells on one hand, and nanotechnology applied to biomaterials on the other. The combination of controlled stem cell fate with advanced nanostructured scaffolds is a golden opportunity to reach the goal of whole organ regeneration for biomedical use. However, key issues exist for the clinical use of stem cells, such as the lack of international standardization of cell handling procedures. This enables private clinics, which offer hope to those suffering from conditions with no conventional therapy, to flourish. Unfortunately, many of these treatments are not supported by experimental evidence, lack regulation and impartial monitoring. Many will remember mediatic cases as infamous examples of "pseudo-science", i.e., a methodology that uses scientific vocabulary but fails to provide evidence based on rigorous protocols and high quality standards [1]. The public is thus confused on the topic and stem cell therapy receives a discredit that is unfair to the scientific effort devoted to this area. In fact, autologous stem cell transplantation is used for some long- and well-established treatments, with clinical trials reporting its beneficial use to ameliorate even challenging conditions affecting heart [2] or nerve [3] tissues. The field is moving so rapidly that the first autologous stem cell medicinal product is already reaching the market: Holoclar ${ }^{\circledR}$ has been conditionally approved in Europe to restore the eyesight of patients with severe cornea damage [4].

Despite such rapid progress in regenerative therapy, unsolved issues remain. The typical procedure for stem cell therapy involves collection of stem cells from a patient, their proliferation to reach suitable numbers to regenerate whole tissues, and their local re-injection in the affected area to be repaired. For this protocol to be successful, stem cells need to be organized and contained within a suitable scaffold. Despite the many commercially available options, there is yet ample scope for improved biomaterials [5]. An alternative strategy that avoids cell passage outside the patient's body requires use of the scaffold alone, which must offer an optimal microenvironment to attract endogenous stem cells from their natural niche towards the site of injury [6]. In either case, in the new paradigm the biomaterial can be seen as a tool to direct stem cell fate, in synergy with, or even as a substitute for, expensive biochemical factors. In principle, this could be achieved through unprecedented nanotopography-, surface adsorption-, and 
chemical profile-mediated mechanisms [5]. The frontiers of knowledge have to be pushed in a concerted multidisciplinary effort, and, especially, we must take advantage of the opportunity offered by working at the nanoscale.

Amongst nanomaterials, carbon nanotubes (CNTs) display a unique set of properties to revolutionize the field $[7,8]$. Mechanical resilience, lightness, elasticity, and nanotopography are key advantages to build a light, durable scaffold. Their optical, thermal, and electronic properties have been recognized for their tremendous potential to realize a qualitative, and timely, progress in the biomedical field [9]. It is not sufficient to exploit their mechanical advantage over conventional materials, as typically reported in literature. Mastering their conductive properties and excellent performance as cell-adhesive substrates make them stand out in the challenge of engineering replacement heart and nerve tissue[10]. Exciting findings are emerging at a fast pace, exploiting CNT unique properties in new and synergistic ways. The elongated morphology of CNTs is proving instrumental for the hierarchical alignment of components into anisotropic biomaterials, tailored to mimic the morphologies of heart and nerve tissues.

Electrospinning of a CNT hybrid hydrogel produces a 3D, conductive scaffold, with nanofiber orientation index matching that of the left ventricular tissue. Such tough, yet flexible, nanostructured and electroactive biomaterial maintains viability of seeded cardiomyocytes and promotes their alignment to achieve synchronous beating [11]. Thermoresponsive CNT-bearing 3D hydrogels prove effective in enhancing the engraftment, survival and differentiation of stem cells in infarcted myocardium [12]. The core of CNT's successful performance in heart engineering is their electrifying effect on cardiomyocytes, which appear more electrically active and better connected to form heart tissue with synchronous beating [13]. Such an effect holds promise to solve the issue of arrhythmias arising from non-conductive scaffolds, as exemplified by a chitosan-based hydrogel where the inserted CNTs act as nanobridges that electrically connect cardiomyocytes [14]. The above-described CNT effects in vivo have been investigated at the level of cell biology to understand which biochemical processes are affected by CNTs. The observed enhancement in the formation of heart tissue with synchronous beating is a consequence of an increase of electrochemical connections (i.e., "junctions") between cells [13]. This fact appears to be the manifestation of integrinmediated biochemical pathways, which are activated by CNTs [15]. The exact biochemical mechanisms underpinning such events are unclear, yet we should remember that integrin proteins (involved in cell adhesion and movement) strongly interact with the cellular machinery that generates motion (e.g., actin and tubulin proteins, which provide the structure that maintains internal cellular organization and mechanical support, or cytoskeleton) [16]. Interestingly, the high aspect ratio of CNTs offers a structural mimic of such elongated proteins, permitting CNTs to participate in supramolecular hybrids that generate cell motion [17],[18]. CNT-cytoskeleton interaction can result in an increase of the ability of cells to migrate (i.e., to move from one location to another). This phenomenon can be explained in terms of augmented production of 1) proteases involved in extracellular matrix (ECM) remodeling (i.e., hydrolyzing the ECM components that anchor cells to a certain position in space) [19] and of 2) cytoskeleton components such as actin protein that allow cells to move [20]. Fine control over these processes would offer a tremendous opportunity to develop "smart" nanostructured scaffolds, e.g. aimed at mobilization of endogenous stem cells.

CNTs could provide advanced scaffolds for artificial neural networks and nerve tissue regeneration because of their known electrifying effect on neurons [21] and ability to render dendritic cells less immunogenic [22], which would address the issue of inflammatory response around implants. In addition, their elongated morphology favors hierarchical nanomaterial assembly, e.g. via nanofiber electrospinning. Electrospun CNT- poly(lactic- co -glycolic acid) composite scaffolds indeed promote electrically actuated neural differentiation of human stem cells [23]. This is not too surprising considering that, CNTs have a similarly electrifying effect on neurons. In other words, in the presence of CNTs, neurons reach more rapidly their functional maturity, including the ability to generate nerve impulses [24]. This is likely the result of electrical "shortcuts" allowed by CNTs that connect proximal and distal compartments of the neuron [25]. However, it is key to select the appropriate method for CNT functionalization for use in tissue engineering. The relevance of the level and nature of covalent modification is emerging just now. Indeed, aminofunctionalized CNTs produced via different routes yield disparate inflammation responses in the brain [26]. Besides, high functionalization levels reduce CNT metallic character, leading to increased resistance and 
loss of CNT boosting effect on neurons. It is thus preferable to maintain a low level of added functional groups to better preserve the conductive properties of CNTs [27]. Preservation of the highly conductive character of CNTs is essential to translate the beneficial effects of CNT-neuron interfacing from 2D to 3D scaffolds, to enhance functional organization and synchronization in neuronal networks [28].

If the challenging objective to control stem cell fate through appropriate nanomaterial guidance in a CNTbased electroactive scaffold is met, then we will be able to regenerate whole organs, including those based on conductive tissue. Stem cells are ideal elements to generate a platform for the 3D-bioprinting of whole organs, since the same printing process can be applied for subsequent cell differentiation into diverse functional tissues. Formulations of CNT-reinforced bio-inks are being optimized and have been also applied to the bioprinting of vascular conduits [29]. Imagine a world where 3D-prints of whole organs, hearts and livers, are one-click away from doctors sitting at their computer desks. Organized tissues could be grown from patients' own cells, avoiding the issue of organ rejection, and organ regeneration would be available to all without the need for transplant waiting lists. 3D-bioprinting companies are making news headlines, and one product standing out is the innovative scaffold-free, liver-tissue patch built from a donor's cells [30].

In conclusion, we provide this concise account of the most recent pioneering work on the use of CNTs in regenerative medicine to illustrate their unique multi-faceted properties to support, connect, and control cell fate, especially towards the 3D printing of artificial organs. Their unique set of versatile electronic, optical, and mechanical properties offer a tremendous opportunity for therapy, especially if combined with powerful resources, such as those offered by stem cells, and advanced technological tools, such as 3Dprinting. It is evident that mastering this complex set of properties is a very demanding task, especially in light of the multidisciplinary knowledge it requires, spanning from biology through chemistry to physics. The purpose of this brief yet broad display of proof of concept studies is to clarify where CNTs stand in the biomaterial field, and why their unique position will unlikely be taken by other nanomaterials. In view of the limited viable options to reconstitute disconnected neural networks or failed organs, it is scientists' responsibility to take the courageous steps to tailor CNTs for their successful adoption in therapy, since this opportunity is not to be missed.

\section{REFERENCES}

[1] E. Cattaneo, G. Corbellini, Nature, 510 (2014) 333-335.

[2] A.W. Heldman, D.L. DiFede, J.E. Fishman, et al., JAMA, 311 (2014) 62-73.

[3] C. Ren, R.-I. Geng, W. Ge, X.-Y. Liu, H. Chen, M.-R. Wan, D.-Q. Geng, Cell Biochem. Biophys., 69 (2014) 179-187.

[4]http://www.ema.europa.eu/ema/index.jsp?curl=pages/medicines/human/medicines/002450/human_m ed_001844.jsp\&mid=WCOb01ac058001d124, last accessed on 25-10-2015.

[5] W.L. Murphy, T.C. McDevitt, A.J. Engler, Nat. Mater., 13 (2014) 547-557.

[6] I.K. Ko, S.J. Lee, A. Atala, J.J. Yoo, Exp. Mol. Med., 45 (2013) e57.

[7] S. Marchesan, M. Melchionna, M. Prato, Fullerenes, Nanotubes, Carbon Nanostruct., 22 (2013) 190-195.

[8] S. Marchesan, M. Prato, ACS Med. Chem. Lett., 4 (2013) 147-149.

[9] S. Marchesan, K. Kostarelos, A. Bianco, M. Prato, Mater. Today, 18 (2015), 12-19.

[10] S. Marchesan, M. Melchionna, M. Prato, ACS Nano, 9 (2015), 9441-9450.

[11] M. Kharaziha, S.R. Shin, M. Nikkhah, S.N. Topkaya, N. Masoumi, N. Annabi, M.R. Dokmeci, A. Khademhosseini, Biomaterials, 35 (2014) 7346-7354.

[12] X. Li, J. Zhou, Z. Liu, J. Chen, S. Lü, H. Sun, J. Li, Q. Lin, B. Yang, C. Duan, M. Xing, C. Wang, Biomaterials, 35 (2014) 5679-5688.

[13] V. Martinelli, G. Cellot, F.M. Toma, C.S. Long, J.H. Caldwell, L. Zentilin, M. Giacca, A. Turco, M. Prato, L. Ballerini, L. Mestroni, ACS Nano, 7 (2013) 5746-5756.

[14] S. Pok, F. Vitale, S.L. Eichmann, O.M. Benavides, M. Pasquali, J.G. Jacot, ACS Nano, 8 (2014) 9822-9832. 
[15] J. Zhou, J. Chen, H. Sun, X. Qiu, Y. Mou, Z. Liu, Y. Zhao, X. Li, Y. Han, C. Duan, R. Tang, C. Wang, W. Zhong, J. Liu, Y. Luo, M. Xing, C. Wang, Sci. Rep., 4 (2014), 3733.

[16] C.G. Gahmberg, S.C. Fagerholm, S.M. Nurmi, T. Chavakis, S. Marchesan, M. Gronholm, Biochim. Biophys. Acta, 1790 (2009) 431-444.

[17] H. Shams, B.D. Holt, S.H. Mahboobi, Z. Jahed, M.F. Islam, K.N. Dahl, M.R. Mofrad, ACS Nano, 8 (2014) 188-197.

[18] C.Z. Dinu, S.S. Bale, G. Zhu, J.S. Dordick, Small, 5 (2009) 310-315.

[19] W. Lohcharoenkal, L. Wang, T.A. Stueckle, C.Z. Dinu, V. Castranova, Y. Liu, Y. Rojanasakul, ACS Nano, 7 (2013) 7711-7723.

[20] L. Ju, G. Zhang, X. Zhang, Z. Jia, X. Gao, Y. Jiang, C. Yan, P.J. Duerksen-Hughes, F.F. Chen, H. Li, X. Zhu, J. Yang, PLoS One, 9 (2014) e84974.

[21] A. Fabbro, M. Prato, L. Ballerini, Adv. Drug Deliv. Rev., 65 (2013) 2034-2044.

[22] A. Aldinucci, A. Turco, T. Biagioli, F.M. Toma, D. Bani, D. Guasti, C. Manuelli, L. Rizzetto, D. Cavalieri, L. Massacesi, T. Mello, D. Scaini, A. Bianco, L. Ballerini, M. Prato, C. Ballerini, Nano Lett., 13 (2013) 6098-6105. [23] J. Landers, J.T. Turner, G. Heden, A.L. Carlson, N.K. Bennett, P.V. Moghe, A.V. Neimark, Adv. Healthc. Mater., 3 (2014) 1745-1752.

[24] A. Fabbro, A. Sucapane, F.M. Toma, E. Calura, L. Rizzetto, C. Carrieri, P. Roncaglia, V. Martinelli, D. Scaini, L. Masten, A. Turco, S. Gustincich, M. Prato, L. Ballerini, PLoS One, 8 (2013) e73621.

[25] G. Cellot, E. Cilia, S. Cipollone, V. Rancic, A. Sucapane, S. Giordani, L. Gambazzi, H. Markram, M. Grandolfo, D. Scaini, F. Gelain, L. Casalis, M. Prato, M. Giugliano, L. Ballerini, Nat. Nanotechnol., 4 (2009) 126-133.

[26] G. Bardi, A. Nunes, L. Gherardini, K. Bates, K.T. Al-Jamal, C. Gaillard, M. Prato, A. Bianco, T. Pizzorusso, K. Kostarelos, PLoS One, 8 (2013), e80964.

[27] S. Bosi, A. Fabbro, C. Cantarutti, M. Mihajlovic, L. Ballerini, M. Prato, Carbon, 97 (2016) 87-91.

[28] S. Bosi, R. Rauti, J. Laishram, A. Turco, D. Lonardoni, T. Nieus, M. Prato, D. Scaini, L. Ballerini, Sci. Rep., 5 (2015), 9562.

[29] D. Farzaneh, Y. Yin, Z. Yahui, M.D.J. Aribet, A.S. Edward, T.O. Ibrahim, Nanotechnology, 25 (2014) 145101.

[30] M. Vaidya, Nat. Med., 21 (2015) 2. 